\section{Retreatment With Brodalumab Results in Skin Clearance and Improvements in Quality of Life in Patients With Psoriasis After Treatment Interruption}

\section{OBJECTIVE}

- To present efficacy and health-related quality of life data after brodalumab withdrawal and retreatment

\section{CONCLUSIONS}

- Reinitiation with brodalumab after treatment withdrawal led to robust levels of skin clearance recapture; patients returned to their previous levels of responses by 24 weeks

- Improvement in quality of life was maintained after retreatment with brodalumab, regardless of exposure to prior biologic treatment

- These results are relevant to real-life practice given that it is relatively common for patients to stop and restart their medications because of various factors
April Armstrong,' Brian Keegan, ${ }^{2}$ George Han, ${ }^{3}$ Abby Jacobson

University of Southern California, Los Angeles, CA; ${ }^{2}$ Princeton Dermatology Associates, Monroe, Nj; ${ }^{3}$ Department of Dermatology at Mount Sinai Beth Israel, New York, NY; "Ortho Dermatologics (a division of Bausch Health US, LLC), Bridgewater, NJ

\section{SYNOPSIS}

Psoriasis is a chronic inflammatory skin disease that negatively impacts quality of life, leading to significant physical and emotional burden

Brodalumab is a fully human interleukin-17 receptor $\mathrm{A}$ antagonist approved for the treatment of moderate-to-severe plaque psoriasis in adult patients with inadequate response or loss of response to other systemic therapies ${ }^{2}$

- Treatment interruption is a common real-world experience in individuals with psoriasis'

\section{METHODS}

In a double-blind, placebo-controlled study (NCT0 I708590;AMAGINE-I), ${ }^{4}$ patients with moderato-coserama

- At week 12, patients receiving brodalumab who achieved a static physician's global assessment (SPGA) of 0 or I were rerandomized to their induction dose of

or placebo

Beginning at week 16 , all rerandomized patients who experienced return of disease
( $\mathrm{PGA} \geq 3$ ) qualified for retreatment and received an induction dose of brodalumab Efficacy was assessed by observed psoriasis area and severity index (PASI) response Health-related quality of life was evaluated with the dermatology life quality index (DLQI) in the retreatment group ( $N=79$ ) using nonresponder imputation (NRI), stratified by disease response to previous biologic treatment before entering the
study (prior biologic failure $[\mathrm{n}=\mid 8]$; prior biologic nonfailure $[\mathrm{n}=6 \mid]$ )

\section{RESULTS}

Efficacy

A total of 79 patients randomized to brodalumab $210 \mathrm{mg}$ Q2W in the induction phase and rerandomized to placebo in the withdrawal phase experienced return of disease (retreatment group)

- Most patients with psoriasis who experienced a return of disease after brodalumab withdrawal returned to their previous levels of PASI response after 24 weeks of retreatment with brodalumab (Figure I)

Health-related quality of life

Mean (SE) DLQI scores in patients in the brodalumab retreatment population at baseline (prior biologic fallure, 14.1 [1.9]; prior biologic nonfallure, $2.9[0.8])$ $1.8[0.4]$ ), similar to DLQI scores achieved during the induction phase at week 12 (Figure 2A)

Change in DLQI from baseline to week 52 for the prior biologic failure and nonfailure subgroups were - 11.5
respectively (Figure 2B)

No significant differences in DLQI were observed by prior biologic response through
week 52 ( $P>0.05$ between subgroups)
Figure 1. PASI 75, PASI 90, and PASI 100 rates after 24 weeks of retreatment with

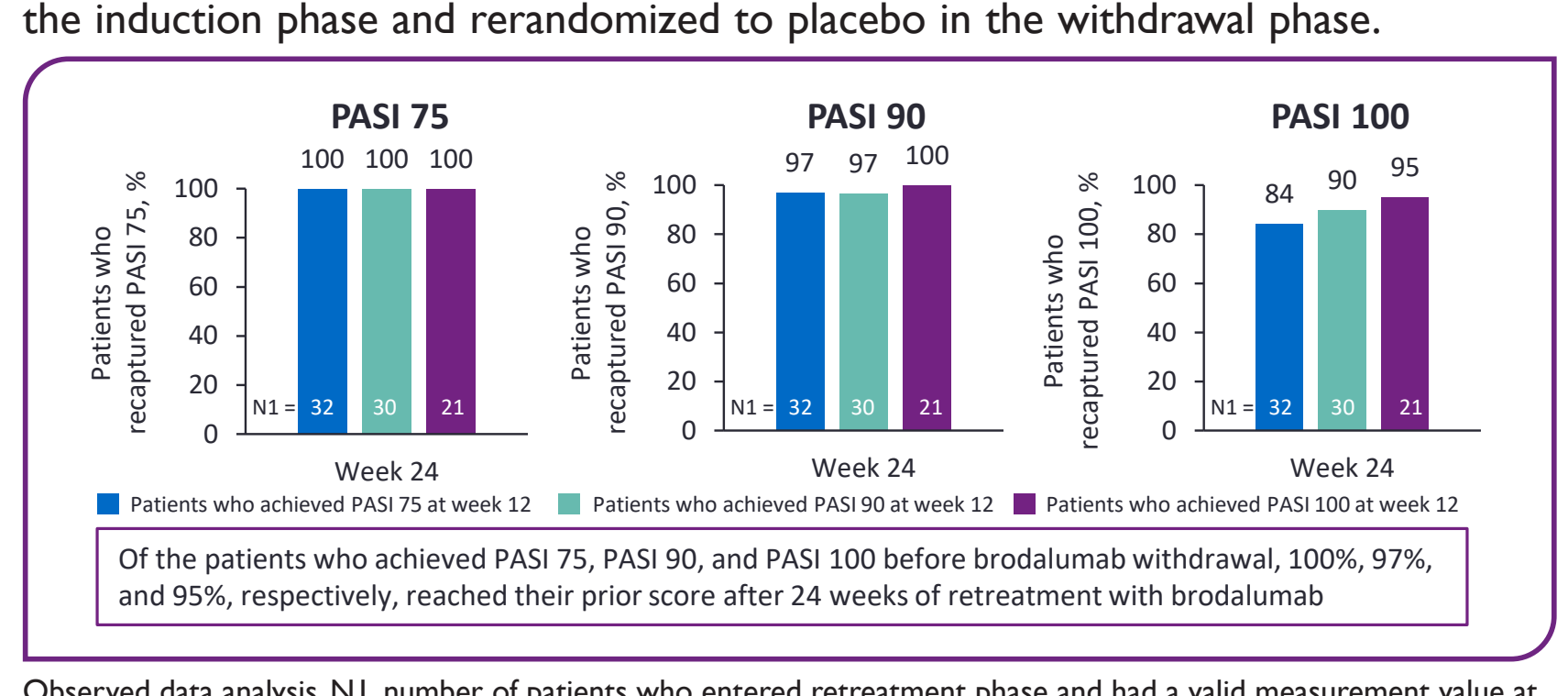

Observed data analysis. NI, number of patients who entered retreatment phase and had a valid measurement value at
the specified week while in the retreatment phase; $\mathrm{PASI} 75,90$, and 100 , psoriasis area and severity index $75 \%$, 90\%, and

Figure 2. (A) Mean total DLQI and (B) change in DLQI from baseline to week 52 for patients in the brodalumab retreatment group by response to prior biologic

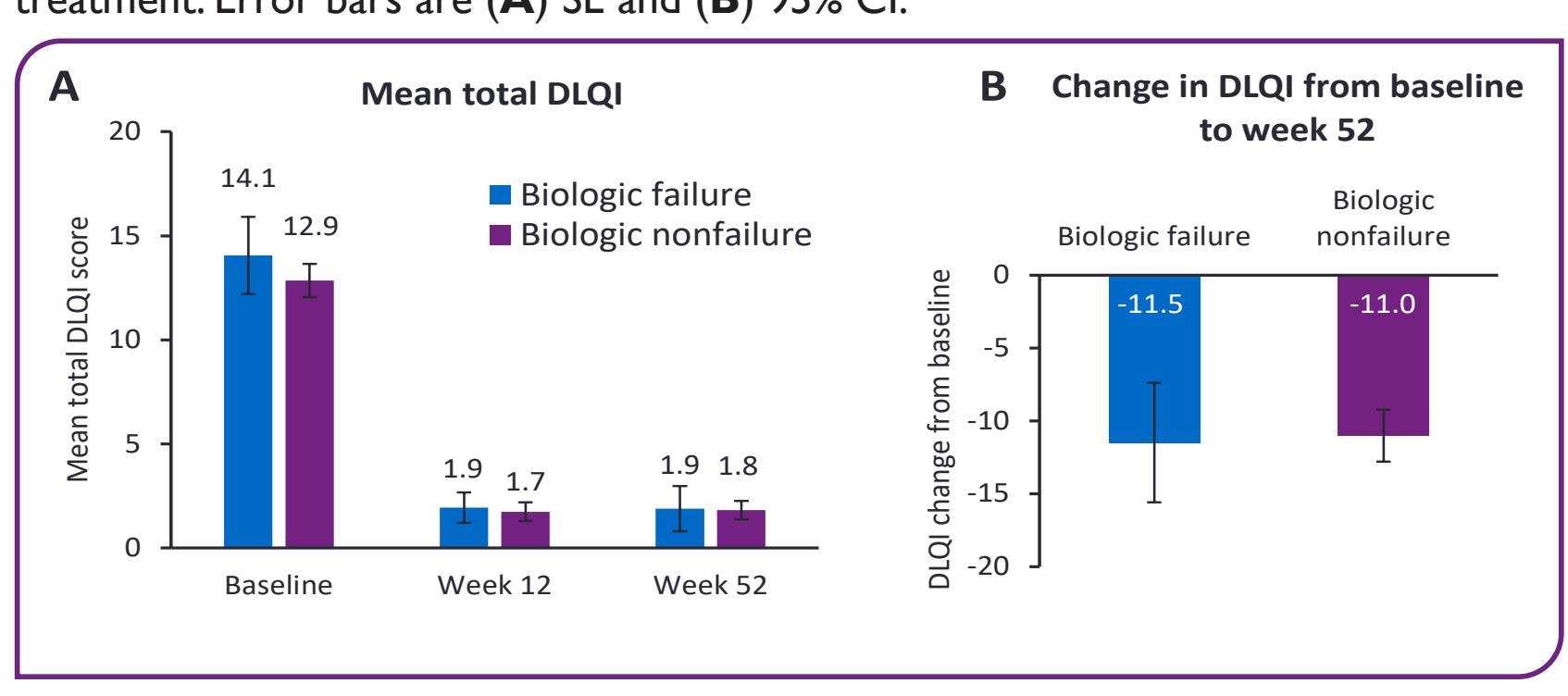

(imputation. DLQI, dermatology life quality index

Funding: This study was sponsored by Ortho Dermatologics. Medical writing support was pre
funded by Ortho Dermatologics. Ortho Dermatologics is a division of Bausch Healt US, LCC

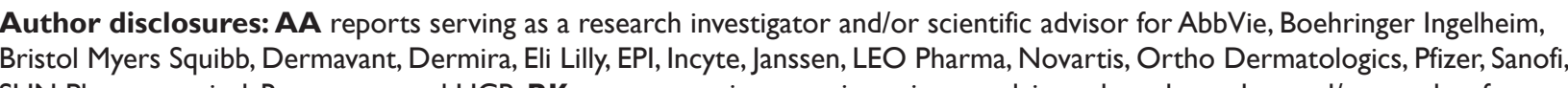

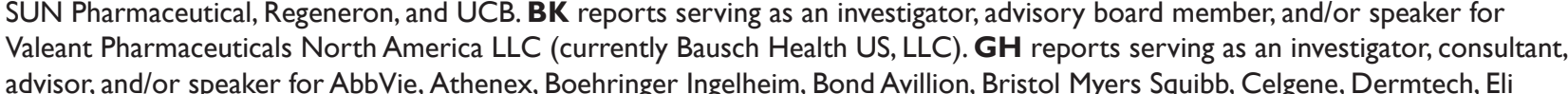

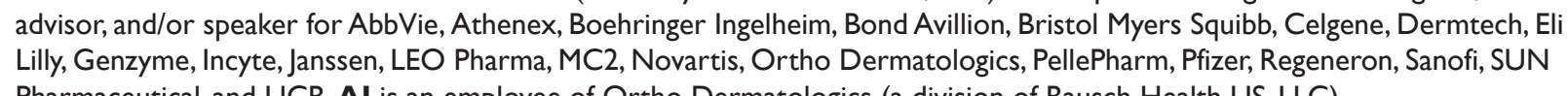
Previous presentation information: Data included in this poster have been previously presented in part at the 44th Annual
Hawaii Dermatology Seminar'; February $16-21,2020$; Maui, $H$.

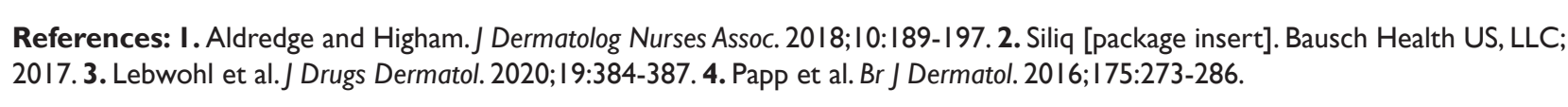

